Tatjana Janzen, Robin Fingerhut, Matthias Heinen, Andreas Köster, Y. Mauricio Muñoz-Muñoz, Jadran Vrabec

\title{
Molecular Modeling and Simulation: Force Field Development, Evaporation Processes and Thermophysical Properties of Mixtures
}

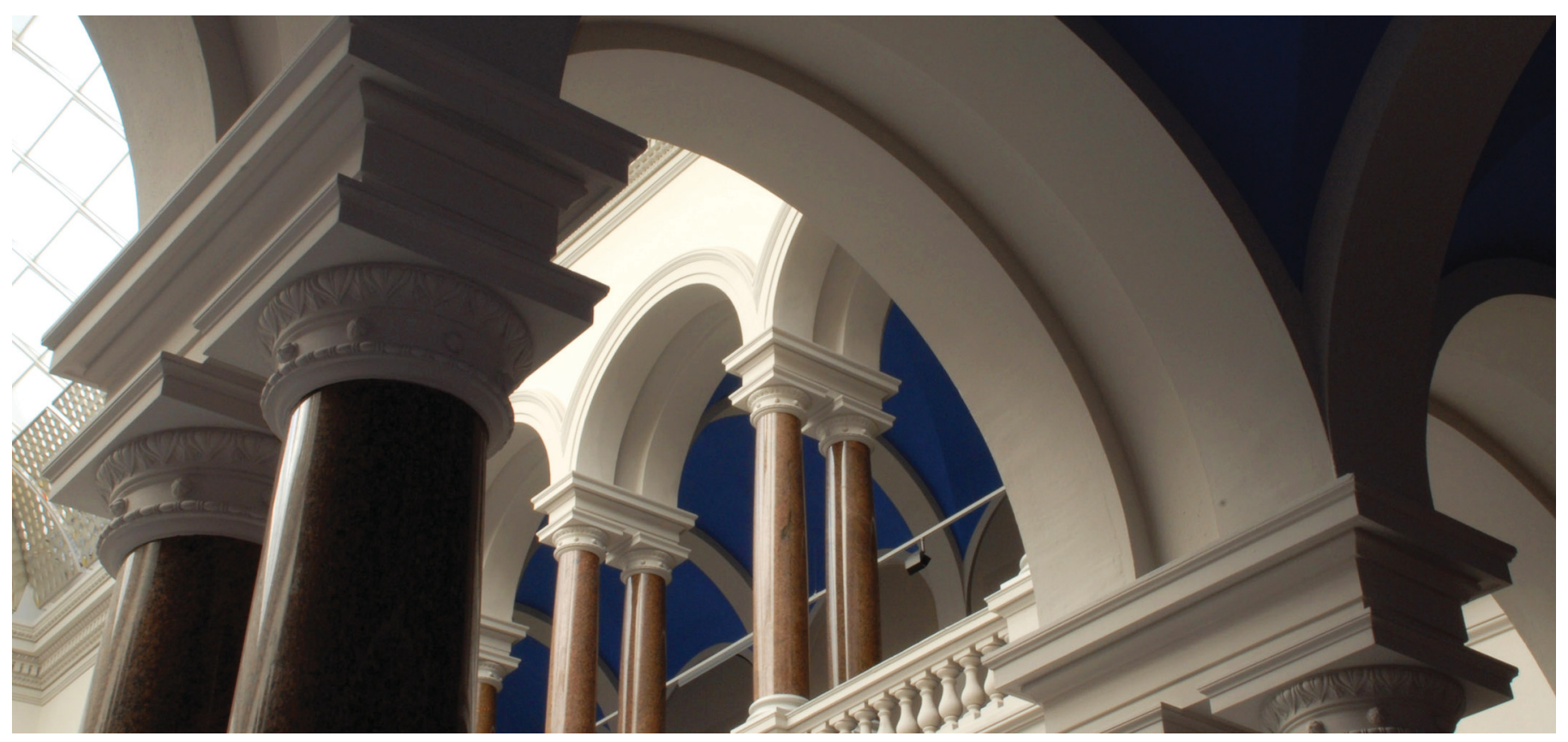

Janzen, T., Fingerhut, R., Heinen, M., Köster, A., Muñoz-Muñoz, Y. M., \& Vrabec, J. (2019). Molecular Modeling and Simulation: Force Field Development, Evaporation Processes and Thermophysical Properties of Mixtures. In High Performance Computing in Science and Engineering ' 18 (pp. 457-474). Springer International Publishing. https://doi.org/10.1007/978-3-030-13325-2_29 


\title{
Molecular Modeling and Simulation: Force Field Development, Evaporation Processes and Thermophysical Properties of Mixtures
}

\author{
Tatjana Janzen, Robin Fingerhut, Matthias Heinen, Andreas Köster, Y. Mauricio \\ Muñoz-Muñoz, and Jadran Vrabec
}

\begin{abstract}
To gain physical insight into the behavior of fluids on a microscopic level as well as to broaden the data base for thermophysical properties especially for mixtures, molecular modeling and simulation is utilized in this work. Various methods and applications are discussed, including a procedure for the development of new force field models. The evaporation of liquid nitrogen into a supercritical hydrogen atmosphere is presented as an example for large scale molecular dynamics simulation. System size dependence and scaling behavior are discussed in the context of Kirkwood-Buff integration. Further, results for thermophysical mixture properties are presented, i.e. the Henry's law constant of aqueous systems and diffusion coefficients of a ternary mixture.
\end{abstract}

\section{Introduction}

Molecular modeling and simulation provides a broad range of possibilities for the investigation of various thermophysical properties. On the one hand, it is a powerful tool for the prediction of thermodynamic data of pure fluids as well as mixtures, which are needed for process design in chemical and energy technology engineering. Especially when dealing with toxic substances or extreme conditions, challenging and expensive experiments can be avoided with this approach. On the other hand, molecular simulation not only contributes to the availability of thermodynamic data, but also allows for a precise insight into the fluid behavior on the microscopic level that governs macroscopic behavior. This promotes the understanding of different phenomena and yields a connection between microscopic structure and thermodynamic properties.

T. Janzen $\cdot$ R. Fingerhut $\cdot$ M. Heinen $\cdot$ A. Köster $\cdot$ Y. M. Muñoz-Muñoz $\cdot$ J. Vrabec Thermodynamics and Thermal Separation Processes, Technical University Berlin, Ernst-ReuterPlatz 1, 10587 Berlin, Germany, e-mail: vrabec@tu-berlin.de 
To exploit the possibilities of molecular modeling and simulation, suitable computational methods combined with high-performance computing are essential. Within this project, two simulation packages are constantly improved and extended: $m s 2[1,2,3]$, for equilibrium simulations of bulk fluids, and $l s 1$ mardyn [4], for the simulation of non-equilibrium systems and flow phenomena on the nanoscale. Several exemplary application cases of these two software packages are presented in this work, including model development and molecular simulations of varying system sizes and different thermodynamic properties of mixtures.

\section{Force Fields for Cyclohexylamine, Aniline and Phenol}

The reliable representation of thermodynamic data by molecular simulation is determined by the quality of the underlying force field, i.e. the mathematical representation of molecular configurational energy $U$. Numerous studies have been carried out on this matter, e.g. using sophisticated numerical methods, such as machine learning [5, 6] or by combining ab initio and classical molecular simulations [7].

The computational effort of classical molecular simulations for systems composed of small molecules may be reduced by neglecting internal molecular degrees of freedom and assuming pairwise additive interactions, i.e. taking the configurational energy as $U=\sum_{i=1}^{N} \sum_{j>i}^{N-1} U_{i j}=U_{\text {elec }}+U_{\text {disp }}$, wherein $N$ is the number of particles of the system and $U_{i j}$ represents the interaction energy between particles $i$ and $j$. It depends on the relative position of the molecular interaction sites belonging to molecules $i$ and $j$ and their electrostatic interaction parameters.

According to this approach, a force field for classical molecular simulations possesses three types of parameters. The electrostatic and geometric parameters as well as the dispersion energy parameters. Molecular geometry, i.e. the location of each atom in the molecular framework, and the electrostatic parameters can be inherited from ab initio calculations, e.g. using the Møller-Plesset 2 method. The use of ab initio simulations is the backbone for developing force fields and the initial starting point of our developments as can be seen in Fig. 1.

On the other hand, the intermolecular dispersion energy $U_{\text {disp }}$ can be described by the Lennard-Jones (LJ) potential, i.e. $U_{\text {disp }}=U_{\text {disp }}(\varepsilon, \sigma)$, where $\varepsilon$ and $\sigma$ are fitting parameters, often located at the position of each atom. Computational effort can be reduced even more by using the united-atom approach, which consists of grouping several atoms and assigning them a common center of force, represented by a single set of LJ parameters $\varepsilon$ and $\sigma$. Indeed, the selection of the parameters to be fitted is a matter of technical art. Once the LJ parameters to be adjusted are selected, the optimization can be carried out following the workflow presented in Fig. 1. Molecular Monte-Carlo (MC) or molecular dynamics (MD) simulations are carried out for calculating the density of the pure fluid. Subsequently, vapor-liquid equilibrium (VLE) simulations are carried out with the Grand Equilibrium method, which requires the chemical potential values. 


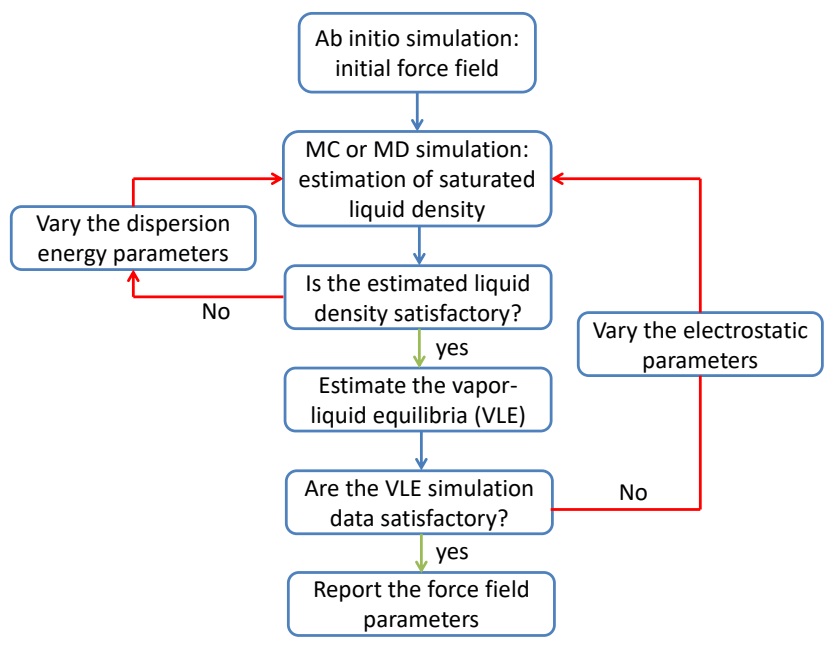

Fig. 1 Flowchart for developing multi-site LJ + point charges force fields.

The workflow given in Fig. 1 was employed for developing three new multi $\mathrm{LJ}$ site + point charge force fields for cyclohexylamine $\left(\mathrm{C}_{6} \mathrm{H}_{13} \mathrm{~N}\right)$, aniline $\left(\mathrm{C}_{6} \mathrm{H}_{7} \mathrm{~N}\right)$ and phenol $\left(\mathrm{C}_{6} \mathrm{H}_{6} \mathrm{O}\right)$. Cyclohexylamine, being technically used e.g. to delay the degradation of rubber or to prevent corrosion in steam plants, was represented by seven LJ sites and four point charges. Two of those point charges are positive and located at hydrogen atom positions, shown in white on top of Fig. 2. One negative charge is located at the center of the nitrogen atom, shown in blue on top of Fig. 2 and the last positive charge is located at the center of the united atom methanetriyl LJ site, depicted in orange. Sites in yellow on top of Fig. 2 stand for the five identical methanediyl LJ united-atom groups of cyclohexylamine, whose parameters were selected to be fitted.

Aniline, important e.g for the pharmaceutic industry, was represented by seven LJ sites and four point charges. Similarly to cyclohexylamine, three point charges are located at the amine group. Two positive charges are at the center of the hydrogen atoms, shown in white on top of Fig. 2. One negative point charge is located at the center of the nitrogen LJ site, shown in blue on top of Fig. 2 and the last positive charge is located at the center of the carbon $\mathrm{LJ}$ site, depicted in black. The sites in orange represent the five methine united-atom LJ sites forming the aromatic ring of the aniline molecule. The aromatic ring of phenol, which is important to yield phenol-formaldehyde resins, is similarly represented as the aniline one. The hydroxyl group, bonded to the aromatic ring, is represented by three point charges. One positive charge is located at the center of the hydrogen atom, one negative charge is located at the center of the oxygen LJ site, which is shown in red on top of Fig. 2. The LJ parameters of the methine sites in both aromatic rings were selected to be fitted to experimental VLE data. 


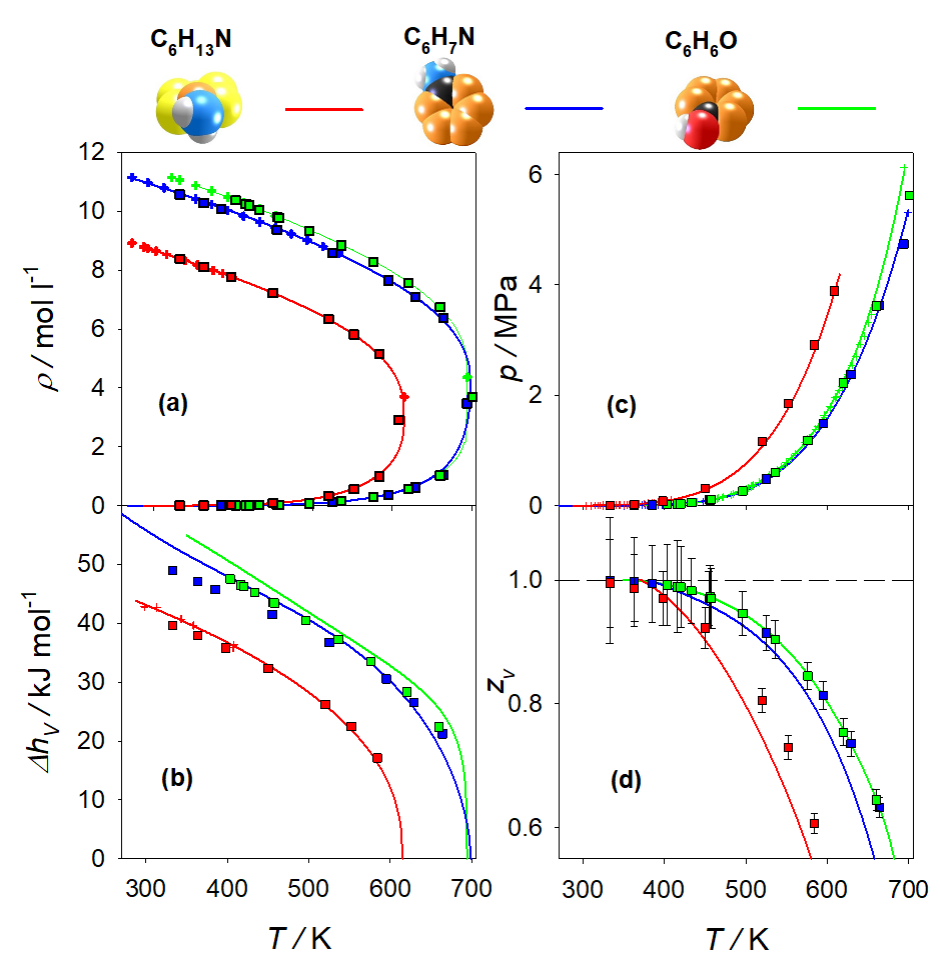

Fig. 2 Vapor-liquid equilibrium results for cyclohexylamine (red symbols), aniline (blue symbols) and phenol (green symbols). Panel (a) represents the saturated liquid and vapor density results, panels (b) and (c) stand for the enthalpy of vaporization and vapor pressure, respectively, and panel (d) shows the compressibility factor of the saturated vapor phase. Lines represent DIPPR correlations, crosses are the available experimental literature data and squares are present molecular simulation results.

Fig. 2 gives an overview on the present molecular simulation results for these three important fluids. These data are in good agreement with experimental literature values and the DIPPR correlations. The absolute average deviations for phenol with respect the DIPPR correlations are $3.5 \%$ for vapor pressure, $0.3 \%$ for saturated liquid density and $5.6 \%$ for enthalpy of vaporization, considering the temperature range where experimental data are available. The cyclohexylamine force field exhibits absolute average deviations of $4.7 \%$ for vapor pressure, $0.1 \%$ for saturated liquid density and $3.3 \%$ for enthalpy of vaporization. The absolute average deviations for aniline are $6.7 \%$ for vapor pressure, $0.4 \%$ for saturated liquid density and $7.2 \%$ for enthalpy of vaporization. Critical data were calculated as well and they are shown in Fig. 2. The critical temperature of phenol was slightly overestimated with respect to literature values, while critical pressure and density were underestimated. The deviations are $1.0 \%,-8.6 \%$ and $-15.3 \%$, respectively. Relative deviations of critical temperature, pressure and density with respect to literature data for cyclohexylamine are $-1.1 \%,-7.1 \%$ and $27 \%$, respectively. Analogously, for aniline these 
numbers are $-0.7 \%,-0.7 \%$ and $-5.9 \%$. Furthermore, present molecular simulation results are consistent with a criterion recently proposed by Nezbeda [8] as can be seen in Panel (c) of Fig. 2.

\section{Stationary Evaporation}

The evaporation of liquid nitrogen into a supercritical hydrogen atmosphere was investigated by large scale molecular dynamics (MD) simulation. This work was motivated by the results of Dahms et al. [9], who studied that injection for two different operating pressures: 1 and $6 \mathrm{MPa}$. In case of the lower pressure, their liquid nitrogen jet disintegrated into small droplets forming a spray, whereas in case of the higher pressure, diffuse mixing occurred. Dahms et al. used nitrogen as a safe substitute for oxygen in their experiments, which were aimed at the optimization of combustion processes in rocket engines. It is well known that the interface between liquid and vapor phase plays a key role in evaporation processes. Fundamental investigations of evaporation into vacuum based on model liquids show that the magnitude of the evaporation flux is strongly dependent on the interface temperature. With decreasing interface temperature, the evaporation flux drops down significantly [10]. The interface properties, especially the temperature, are thus decisive for evaporation dynamics. Consequentially, it was focused in the present work on the interfacial region between liquid nitrogen and the high pressure hydrogen atmosphere. Because of its sound physical basis and atomistic resolution, MD simulation is an appropriate approach to obtain a better understanding of the findings by Dahms et al. [9].

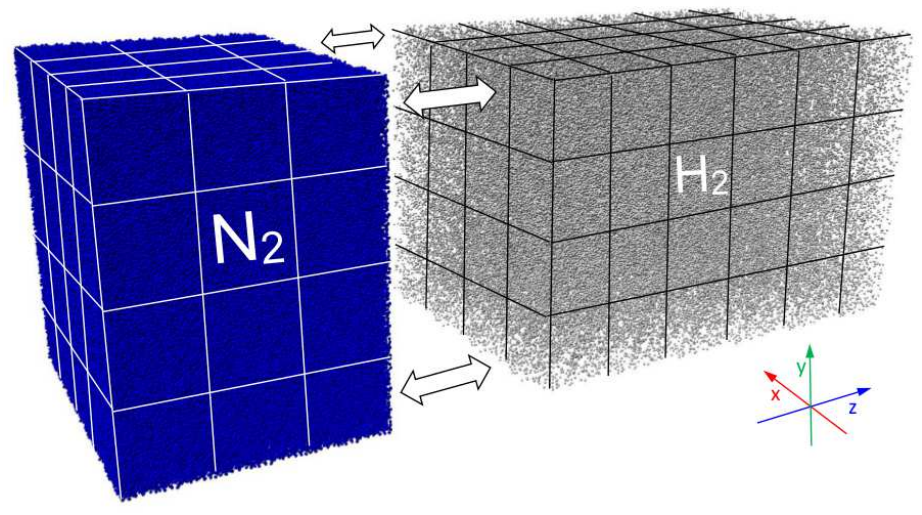

Fig. 3 Schematic view of system construction by the ReplicaGenerator module. The liquid nitrogen $\mathrm{N}_{2}$ phase and the supercritical hydrogen $\mathrm{H}_{2}$ phase were prepared from small fluid cubes (indicated by grid cells). These small systems were replicated to yield two larger homogeneous phases. These were equilibrated for an additional short time period and then merged together to yield the initial configuration where the two phases are in physical contact for non-equilibrium molecular dynamics simulations (NEMD). 
Molecular systems including an overall number of $\approx 6 \cdot 10^{6}$ molecules were set up with a planar interface, with an extent of $A_{x y}=85 \times 85 \mathrm{~nm}^{2}$, between a liquid nitrogen film and a supercritical hydrogen atmosphere. A schematic view of how the system was constructed is depicted in Fig. 3. A new module of the MD code ls1 mardyn [4], namely the ReplicaGenerator, was used to create comparatively large homogeneous phases of pure nitrogen $\mathrm{N}_{2}$ and pure hydrogen $\mathrm{H}_{2}$ out of small ones. First, the small systems were equilibrated to obtain physical molecular configurations. Subsequently, they were replicated and merged together, yielding larger phases. These were equilibrated again for a short time until the replication pattern vanished. This procedure reduces the equilibration time which is particularly important for large phases.

The system was delimited on both left and right sides ( $z$ direction) by reflective boundaries, spanning a system width of $L_{z}=190 \mathrm{~nm}$. These boundaries were extended by additional molecules with fixed spatial positions to reduce their influence. To mimic an infinitely extended interface, periodic boundary conditions were specified in $x$ and $y$ directions. The temperature of the liquid nitrogen of $T_{\mathrm{N}_{2}}=118 \mathrm{~K}$ was controlled within a control volume on the left side, which had a width of about ten molecule diameters only. The temperature of hydrogen was controlled within a control volume on the right side to be $T_{\mathrm{H}_{2}}=270 \mathrm{~K}$, also maintaining a constant density and composition. The latter was achieved by converting all incoming nitrogen molecules to hydrogen molecules such that a mole fraction of $y_{\mathrm{H}_{2}}=1$ (pure hydrogen) was maintained. To establish a stationary evaporation process, a liquid reservoir added new nitrogen molecules over the left boundary of the control volume into the liquid phase. The feed rate of the reservoir was adjusted to balance the rate of evaporated nitrogen molecules entering the control volume in the gas phase. There were no interventions between the control volumes, except for the numerical solution of Newton's equations of motion of the molecular ensemble with the MD code lsl mardyn [4]. The molecular models for nitrogen and hydrogen were provided by Köster et al. [11], showing a very good agreement with experimental literature data and the Peng-Robinson equation of state for vapor-liquid equilibria of pure substances and mixtures, considering a large temperature and pressure range. A series of three simulations with varying system pressure, controlled by the target density of the hydrogen gas in the right control volume, was carried out: $p=4,12$ and $20 \mathrm{MPa}$. During simulation, various profiles were sampled. Each profile was averaged over $10^{4}$ time steps, with a time step length of $\Delta t=2 \mathrm{fs}$. At the beginning of the simulations, all profiles were step functions because the systems were set up by merging an equilibrated liquid nitrogen phase at a temperature of $T_{\mathrm{N}_{2}}$ with an equilibrated hydrogen gas phase at a temperature of $T_{\mathrm{H}_{2}}$. Within the first nanosecond, these profiles changed rapidly, developing into a smooth transition between the liquid phase and gas phase states maintained by the control volumes. After about $3 \mathrm{~ns}$, the profiles hardly changed any more such that a stationary process had been attained. Fig. 4 shows the temperature, hydrogen mole fraction and density profiles of the simulation series for two pressure levels $p=4$ and $20 \mathrm{MPa}$ after an elapsed time of 0,1 and $3 \mathrm{~ns}$. The case of $p=12 \mathrm{MPa}$ showed qualitatively similar results as $p=20 \mathrm{MPa}$ and the results are therefore not illustrated here. Looking at 
the right column of Fig. 4, several aspects can be seen that may contribute to the understanding of the macroscopic system studied by experiment [9]. The density profile of the low pressure case ( $p=4 \mathrm{MPa}$ ) has a comparatively sharp interface, transitioning from the liquid density down to the gas density. The density profiles of the high pressure case ( $p=20 \mathrm{MPa}$ ) exhibit a slightly higher liquid density and a significantly higher gas density due to compression. Moreover, a much broader and smoother transition between the liquid and gas density than in the low pressure case was found.
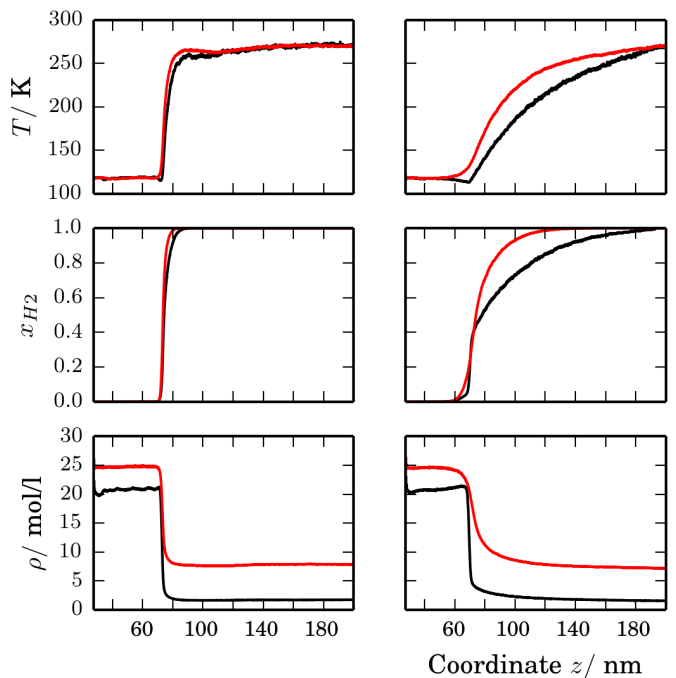
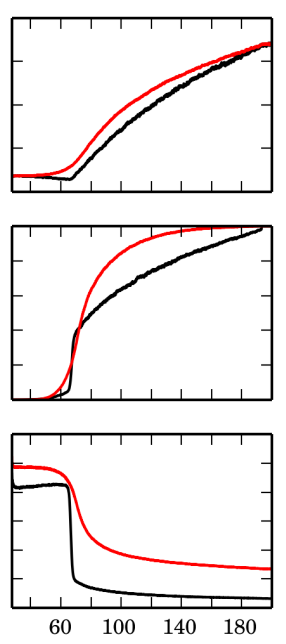

Fig. 4 Temporal evolution of the temperature $T$ (top), mole fraction of hydrogen $x_{\mathrm{H}_{2}}$ (center) and molar density (bottom) profiles of the low pressure case $p=4 \mathrm{MPa}$ (black) and the high pressure case $p=20 \mathrm{MPa}$ (red). Columns left, center, right show profiles after 0,1 and $3 \mathrm{~ns}$.

The temperature profile of the low pressure case shows a minimum within the interface region, indicating that a part of the enthalpy of evaporation is provided by the liquid phase that consequently cools down. From a molecular perspective, the liquid film cools down at the interface because molecules with higher kinetic energy preferentially leave the interface, whereas molecules with lower kinetic energy preferentially remain inside the liquid. The interface temperature decrease is only $3.5 \mathrm{~K}$ because heat is transported from both phases to the interface via temperature gradients. The temperature profiles of the higher pressure cases do not exhibit a temperature minimum at the interface. This can be explained by the higher pressure (and density) of the gas phase, leading to a much stronger thermal coupling with the liquid. Consequentially, a sufficient amount of heat is transported from the hot gas phase to balance the amount of energy taken up by evaporating nitrogen molecules. Instead, the liquid is even heated up, indicating that more energy is transported from 
the gas to the liquid phase than required to drive evaporation. The mole fraction profiles show expected trends: The gas phase is rich in hydrogen and the liquid phase is rich in nitrogen. Consistent with the density profiles, the low pressure case exhibits a sharp transition, whereas the higher pressure cases have a smooth and much broader transition, indicating diffuse mixing.

\section{Kirkwood-Buff Integration}

Local density fluctuations within closed systems can be sampled with KirkwoodBuff integration (KBI) [14] and lead to macroscopic thermodynamic properties. Many-body molecular simulation naturally provides detailed information on the microscopic structure of fluids by means of radial distribution functions (RDF) $g_{i j}(r)$, which are closely related to what is known as local composition. In case of a mixture containing components $i$ and $j$, they are given by

$$
g_{i j}(r)=\frac{\rho_{L, i}(r)}{\rho_{j}}
$$

where the local density of component $i$ in a spherical shell centered around species $j$ is

$$
\rho_{L, i}(r)=\frac{\mathrm{d} N_{i}}{4 \pi r^{2} \mathrm{~d} r},
$$

and $\mathrm{d} N_{i}$ denotes the number of particles of component $i$ therein. The overall partial density is simply $\rho_{j}=x_{j} \rho$. A binary mixture of components 1 and 2 has thus three independent radial distribution functions, i.e. $g_{11}(r), g_{22}(r)$ and $g_{12}(r)=g_{21}(r)$. Moreover, radial distribution functions provide access to the composition dependence of the excess Gibbs energy by means of KBI. A good convergence of their integrals

$$
G_{i j}=4 \pi \int_{0}^{\infty}\left[g_{i j}(r)-1\right] r^{2} \mathrm{~d} r
$$

can be achieved by canonic $N V T$ ensemble simulations employing the formalism of Krüger et al. [16]. The Kirkwood-Buff integrals $G_{i j}$ lead to several macroscopic properties, e.g. the mole fraction derivative of the activity coefficient $\gamma_{1}$ of component 1 is given by

$$
\left(\frac{\partial \ln \gamma_{1}}{\partial x_{1}}\right)_{T, p}=-\frac{x_{2} \rho\left(G_{11}+G_{22}-2 G_{12}\right)}{1+x_{2} \rho x_{1}\left(G_{11}+G_{22}-2 G_{12}\right)} .
$$

For studies on mass transport, the thermodynamic factor $\Gamma$ is vital because it connects the Fick diffusion coefficients $D_{i j}$ and Maxwell-Stefan diffusion coefficients $\bigoplus_{i j}$ (for a binary mixture: $D_{11}=\Gamma_{11} \bigoplus_{12}$ ) and it is given by 


$$
\Gamma_{11}=1+x_{1}\left(\frac{\partial \ln \gamma_{1}}{\partial x_{1}}\right)_{T, p} .
$$

In the field of process engineering, mixture data are of particularly great interest. Properties like partial molar volumes, isothermal compressibility and most interestingly thermodynamic factor can be determined with KBI. To this end, KBI was implemented into our massively-parallel molecular simulation tool ms2 [3]. However, finite system-size effects are challenging for KBI calculations by using molecular simulations of closed systems because KBI is defined for macroscopically large systems only. Further, for large distances $r$, the sampled RDF tend to lose statistical accuracy and may not converge to unity. Accordingly, RDF corrections are crucial [15] in the same way as an extrapolation to macroscopic size [17]. Moreover, it was shown by Galata et al. [12] that simulations with a larger number of particles $N$ may lead to improved results. Thus, parallelization of KBI in $m s 2$ is essential because the computational effort is directly dependent on the number of particles $N$.

Finite system-size effects of KBI were assessed for a binary Lennard-Jones (LJ) mixture for two different system sizes in the closed $N V T$ ensemble with $N=4,000$ and $N=8,000$ particles (LJ parameters $\sigma_{2} / \sigma_{1}=1.5$ and $\varepsilon_{2} / \varepsilon_{1}=0.75$ ). The LJ mixture was studied over the entire molar fraction of $x_{1}=0.05,0.1,0.2, \ldots, 0.95 \mathrm{~mol} / \mathrm{mol}$. The mixture density range was sampled with isobaric-isothermal $N p T$ simulations at constant pressure $p \sigma_{1}^{3} / \varepsilon_{1}=0.03$ and constant temperature $T k_{B} / \varepsilon_{1}=0.85$. Molecular dynamics (MD) NVT simulations for both system sizes were carried out with 800,000 equilibration steps and 15 Mio. production steps with a time step of $0.329 \mathrm{fs}$ and a cutoff radius of $r_{c}=5 \sigma_{1}$. RDF were sampled for each time step with a maximum radius divided into 300 shells. Fig. 5 shows the thermodynamic factor $\Gamma_{11}$ over mole fraction $x_{1}$ for the two system sizes $N=4,000$ and $N=8,000$.

It becomes clear that the simulation results for the thermodynamic factor $\Gamma_{11}$ are slightly improved for larger system sizes because of the decreased deviation to the Wilson model [19] fit. On that point, the thermodynamic factor $\Gamma_{11}$ calculated by the Wilson model may serve for comparison since it is independent from the LJ model error.

The parallelization performance of $m s 2$ was analyzed for the MD NVT ensemble with KBI calculation turned on and off. Therefore, three different system sizes of the above-mentioned LJ mixture with $N=4,000 ; 8,000 ; 16,000$ particles were carried out on CRAY XC40 (Hazel Hen) of HLRS and the runtime of $m s 2$ was measured by changing the number of nodes (each node contained 24 cores). Fig. 6 shows the scaling of MD NVT simulations of $m s 2$ for those three systems with KBI calculation turned on and off.

For all three systems, the cutoff radius was set to $r_{c}=5 \sigma_{1}$ and thus the NVT interaction calculation alone required the same computing power, which only depends on the number of particles $N$ in the cutoff sphere. However, to determine which particles are inside the cutoff sphere, the whole interaction matrix has to be traversed which allows for a computationally effective access to the calculation of RDF shells if KBI is turned on. The computing intensity of traversing the particle matrix is proportional to $N^{2}$. As a result, the computational cost of $m s 2$ should be 


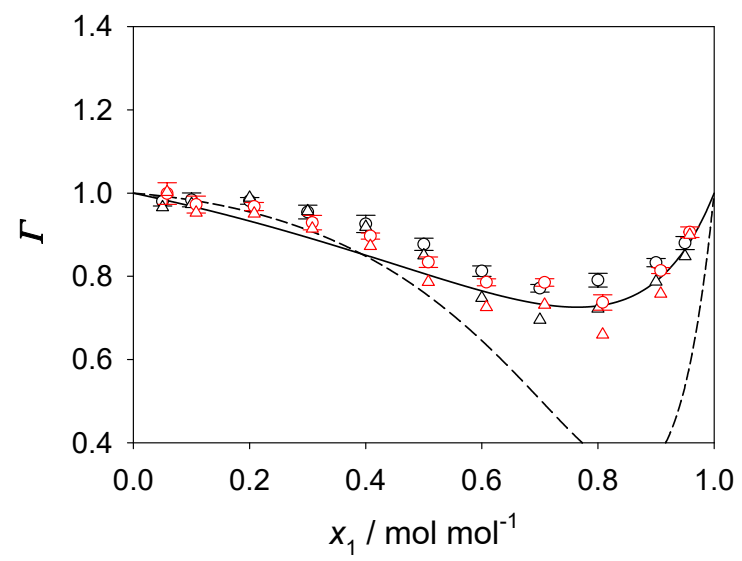

Fig. 5 Thermodynamic factor $\Gamma_{11}$ over mole fraction $x_{1}$ of a binary LJ mixture calculated with $\mathrm{MD}$ and $\mathrm{KBI}$ in the $N V T$ ensemble employing $m s 2$; black symbols: $N=4,000$; red symbols (slighlty shifted to larger $x_{1}$ for visibility reasons): $N=8,000$; circles/triangles: nonextrapolated/extrapolated data to macroscopic system size; solid line: Wilson model [19] fitted to chemical potential data calculated for the LJ mixture with Widom's test particle insertion [18]; dashed line: PC-SAFT equation of state [13].

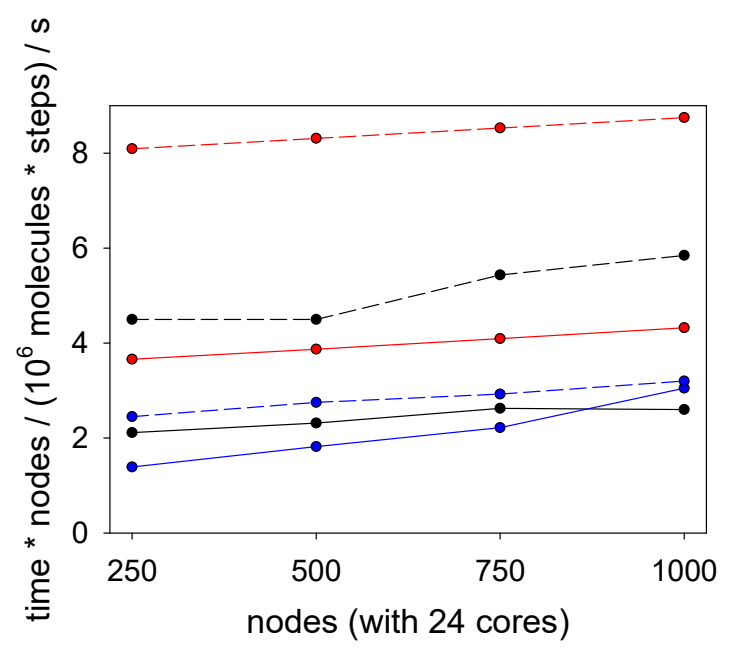

Fig. 6 Strong scaling efficiency of $m s 2$ for a binary LJ mixture in the MD NVT ensemble measured on CRAY XC40 (Hazel Hen); solid line: MD NVT (KBI turned off); dashed line: MD NVT (KBI turned on); blue lines: $N=4,000$; black lines: $N=8,000$; red lines: $N=16,000$ particles. 
in between $N$ to $N^{2}$, e.g. doubling $N$ should lead to a factor 2 to 4 in terms of computational cost. Fig. 6 indicates that doubling the number of particles in $m s 2$ leads to an increase of computational cost smaller than factor 4 if the number of nodes is chosen appropriately so that the overhead is small. Further, the vertical axis gives the computing power (nodes) times computing time per computing intensity (problem size) and thus, a horizontal line would show a perfect strong scaling efficiency of $100 \%$. Consequently, the Fig. 6 points out that $m s 2$ is close to optimal strong scaling, but it of course does not reach $100 \%$ efficiency. Moreover, it becomes clear that for small systems with computationally cheap interaction calculations like LJ mixtures, the strong scaling efficiency decreases with increased computing power (nodes) because of communication overhead.

\section{Henry's law constant}

In an extensive study that was recently published in full [11], the mixture behavior of five substances related to the hydrogen economy, i.e. $\mathrm{H}_{2}$, nitrogen $\left(\mathrm{N}_{2}\right)$, oxygen $\left(\mathrm{O}_{2}\right)$, argon $(\mathrm{Ar})$ and water $\left(\mathrm{H}_{2} \mathrm{O}\right)$, was examined using these techniques. Several thermodynamic properties were considered in this work to describe a substantial number of mixtures, including binary, ternary and quaternary systems. Apart from VLE properties, like vapor pressure, saturated densities or enthalpy of vaporization, also homogeneous density and solubility were studied. The quality of the results was assessed by comparing to available experimental literature data or sophisticated equations of state, showing an excellent agreement in many cases. Consequently, this contribution aims at an improved availability of thermodynamic data that are required for the post-carbon age.

The present work gives a short overview on the Henry's law constant that can be described particularly well with molecular simulation techniques and was used in the work of Köster and Vrabec [11] to assess aqueous systems, i.e. $\mathrm{H}_{2}, \mathrm{~N}_{2}, \mathrm{O}_{2}$ or $\mathrm{Ar}$ in liquid $\mathrm{H}_{2} \mathrm{O}$. Consequently, this property is used in cases where a solute is only little soluble in a solvent. It has to be noted that the purely temperature-dependent definition of the Henry's law constant was considered, wherein the solvent has to be in its saturated liquid state. Therefore, data are presented ranging from the triple point temperature to the critical temperature of $\mathrm{H}_{2} \mathrm{O}$.

The Henry's law constant $H_{i}$ was sampled with the molecular simulation tool $m s 2[1,2,3]$ in the $N p T$ ensemble using 1000 particles in total. The simulation runs typically consisted of $3 \cdot 10^{6} \mathrm{MC}$ cycles, i.e. $2 \cdot 10^{5}$ equilibration and $2.8 \cdot 10^{6}$ production cycles. $H_{i}$ is closely related to the chemical potential at infinite dilution $\mu_{i}^{\infty}[20]$

$$
H_{i}=\rho_{\mathrm{s}} k_{\mathrm{B}} T \exp \left(\mu_{i}^{\infty} /\left(k_{\mathrm{B}} T\right)\right),
$$

wherein $\rho_{\mathrm{s}}$ is the saturated liquid density of the solvent, $T$ the temperature and $k_{\mathrm{B}}$ Boltzmann's constant. An attractive method to calculate the chemical potential at infinite dilution is Widom's test particle insertion [18], where the mole fraction of 
the solute can simply be set to zero such that only test particles are inserted into the pure solvent, i.e. $\mathrm{H}_{2} \mathrm{O}$.

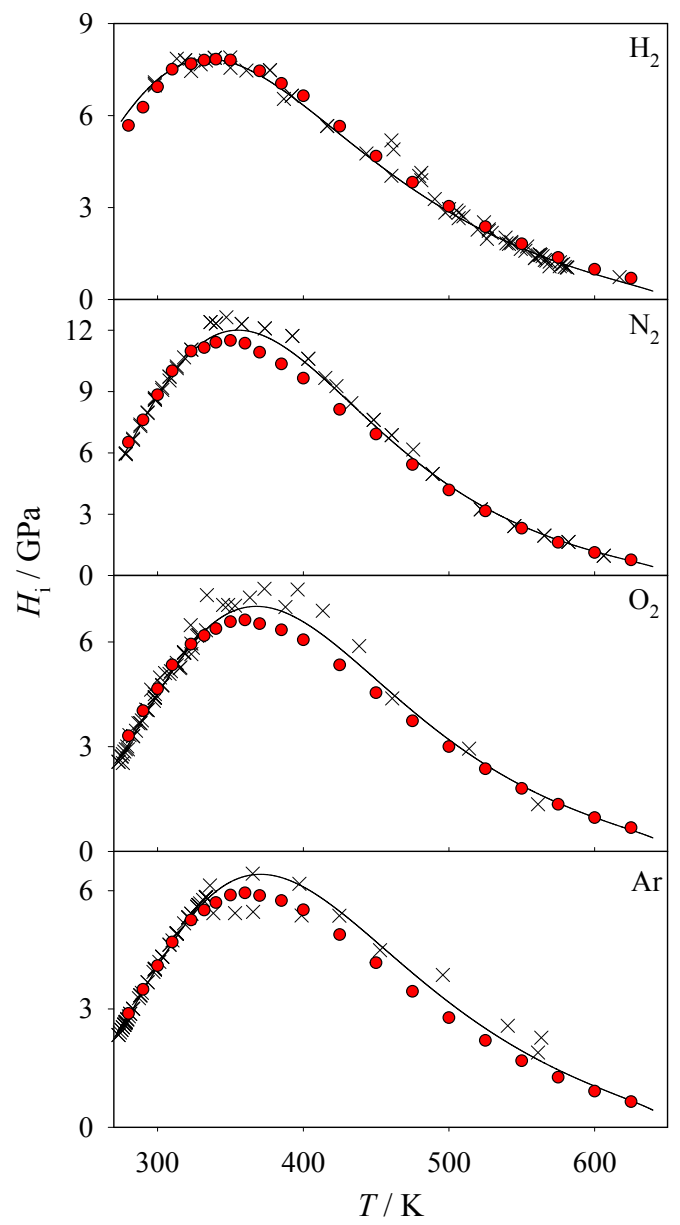

Fig. 7 Henry's law constant of $\mathrm{H}_{2}, \mathrm{~N}_{2}, \mathrm{O}_{2}$ and $\mathrm{Ar}$ in $\mathrm{H}_{2} \mathrm{O}$ (from top to bottom): (•) Molecular simulation results, $(-)$ official IAPWS guideline $[22,21]$ and $(+)$ experimental literature data $\left(\mathrm{H}_{2}\right.$ $\left.[23,24] ; \mathrm{N}_{2}[25,26,24,27] ; \mathrm{O}_{2}[28,29,30,31] ; \operatorname{Ar}[32,33,34,35]\right)$.

All aqueous systems presented in Fig. 7 were validated against experimental data and the official IAPWS correlation [21,22] for the Henry's law constant. They exhibit a strongly non-monotonic behavior that is qualitatively similar for the various solutes and pass through a maximum between 330 and $370 \mathrm{~K}$. Due to the fact that higher values of the Henry's law constant correspond to a low solubility, the solubility decreases with increasing temperature, passes through a minimum and then 
increases again. On the quantitative side, however, large differences between the solutes are present. Near the triple point temperature of $\mathrm{H}_{2} \mathrm{O}$, both $\mathrm{H}_{2}$ and $\mathrm{N}_{2}$ are roughly three times less soluble than $\mathrm{O}_{2}$ and $\mathrm{Ar}\left(H_{\mathrm{H} 2} \approx H_{\mathrm{N} 2} \approx 6 \mathrm{GPa}\right.$ compared to $H_{\mathrm{O} 2} \approx H_{\mathrm{Ar}} \approx 2 \mathrm{GPa}$ ). Moreover, the Henry's law constant values at the maximum differ significantly.

It can be seen that Henry's law constant data were predicted satisfactorily by molecular simulation, especially at temperatures close to the triple and critical point of $\mathrm{H}_{2} \mathrm{O}$. At intermediate temperatures, molecular simulation results for $\mathrm{N}_{2}, \mathrm{O}_{2}$ and Ar deviate slightly, whereas $\mathrm{H}_{2}$ is reproduced almost perfectly. It has to be noted, however, that the force field of $\mathrm{H}_{2}$ was modelled without electrostatic interactions. This was compensated by increasing the unlike LJ interaction energy, resulting in a rather unphysically large value of the binary interaction parameter $\xi=1.52$.

\section{Diffusion Coefficients of Ternary Liquid Mixtures}

Numerous natural and technical processes are determined by transport diffusion of multicomponent liquids, while the underlying microscopic phenomena are still not well understood. Experimental data on multicomponent diffusion coefficients are rather scarce because strong composition dependence and cross diffusion effects aggravate experimental work. Molecular modeling and simulation is a promising route for investigating diffusion coefficients on a microscopic basis to understand the underlying phenomena and to study the relations between intradiffusion and transport diffusion coefficients. Nonetheless, not only the diffusion experiments, but also the simulations are challenging and time consuming. To gain an overall picture of the diffusion behavior of a ternary mixtures, a sufficient amount of state points with varying composition must be studied, also considering the binary subsystems. Thus, sufficient parallelization of the utilized software as well as efficient data handling are essential to overcome this task.

Here, equilibrium molecular dynamics (MD) simulations and the Green-Kubo formalism were used to sample intradiffusion and Maxwell-Stefan (MS) diffusion coefficients of binary and ternary liquid mixtures. Intradiffusion coefficients $D_{i}$, which describe the mobility of molecules of species $i$ in the absence of driving force gradients, were obtained from the time integral of the single particle velocity autocorrelation function averaged over all $N_{i}$ particles belonging to that species [36]

$$
D_{i}=\frac{1}{3 N_{i}} \int_{0}^{\infty}\left\langle\sum_{k=1}^{N_{i}} \mathbf{v}_{i}^{k}(0) \cdot \mathbf{v}_{i}^{k}(t)\right\rangle \mathrm{d} t .
$$

MS diffusion coefficients, which describe mass transport driven by a chemical potential gradient, are directly related to kinetic coefficients which can be sampled from net velocity correlation functions of species $i$ and $j$ [37] 


$$
\Lambda_{i j}=\frac{1}{3 N} \int_{0}^{\infty}\left\langle\sum_{k=1}^{N_{i}} \mathbf{v}_{i}^{k}(0) \cdot \sum_{l=1}^{N_{j}} \mathbf{v}_{j}^{l}(t)\right\rangle \mathrm{d} t .
$$

Fick diffusion coefficients are related to gradients of composition variables and can thus be measured experimentally. For the transformation of MS to Fick diffusion coefficients the thermodynamic factor is needed [42], which can be calculated with excess Gibbs energy $\left(g^{E}\right)$ models that are typically fitted to experimental vaporliquid equilibrium data. Details on the relations between the different coefficients for ternary mixtures can be found in recent publications of our group [38, 39].

One of the ternary mixtures studied within this project was acetone + benzene + methanol. This mixture was investigated at ambient conditions of temperature and pressure, for which experimental data of Fick diffusion coefficients are available in the literature [41]. Diffusion and other transport properties of the binary subsystems were already predicted by MD simulations in the present MMHBF project [40]. The utilized molecular force field models are rigid, united-atom Lenard-Jones type models with superimposed point charges, dipoles and quadrupoles. These models were parametrized using experimental data of the pure components only, thus the mixture behavior was obtained in a strictly predictive manner. MD simulations were carried out with 4000 molecules in a cubic volume over 4 to $5 \times 10^{7}$ time steps of $\sim 1.02$ fs. Further details on the simulation setup used to sample ternary diffusion coefficients can be found in the supplementary material of Ref. [39].

Fig. 8 shows the intradiffusion coefficients $D_{i}$ of the three components over the entire composition range of the ternary mixture acetone + benzene + methanol. These figures are based on simulations for 52 ternary and 40 binary state points at different compositions indicated by points within the graphs. The coefficients of all three components have a similar composition dependence, with lowest values at small acetone content and highest values at high acetone content. Because methanol molecules form hydrogen bonds and often propagate as assemblies, the intradiffusion coefficient is similar to that of benzene. Only at low methanol concentrations this intradiffusion coefficient is significantly higher due to the breaking of hydrogen bonds.

Fig. 9 shows the three MS diffusion coefficients $\bigoplus_{i j}$ of the ternary mixture acetone + benzene + methanol. While the values of the MS coefficient between acetone and benzene $\bigoplus_{12}$ are similar to the intradiffusion coefficients, the other two MS coefficients $\bigoplus_{13}$ and $\bigoplus_{23}$ show a significantly non-ideal behavior. This is also a result of the hydrogen bonding behavior of methanol molecules, which leads to microscopic heterogeneity in mixtures with other fluids and affects their kinetics through correlated propagation. Fick diffusion coefficients predicted from the simulated MS diffusion coefficients combined with the thermodynamic factor calculated with the Wilson $g^{E}$ model are in excellent agreement with experimental data [39]. 

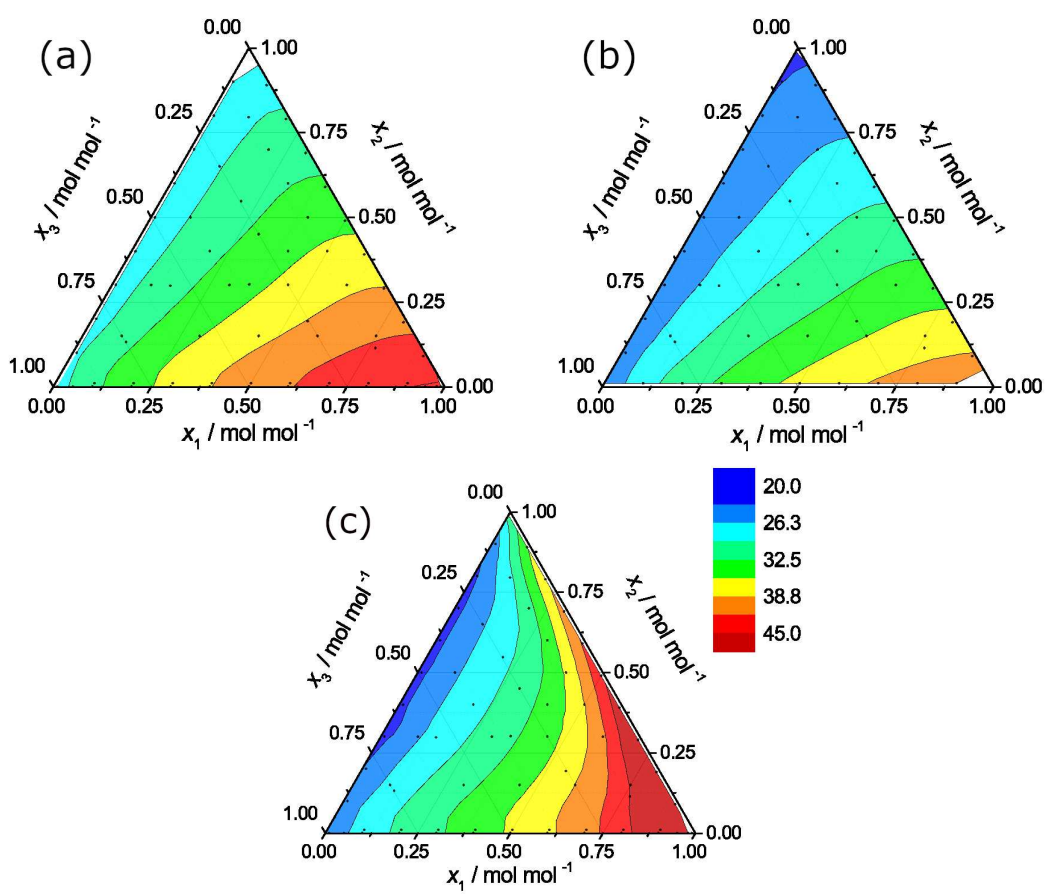

Fig. 8 Intradiffusion coefficient $D_{i}$ (in $10^{-10} \mathrm{~m}^{2} \mathrm{~s}^{-1}$ ) of acetone (a), benzene (b) and methanol (c) in their ternary mixture at $T=298.15 \mathrm{~K}$ and $p=0.1 \mathrm{MPa}$.

\section{Conclusion}

Several applications of molecular modeling and simulation were outlined in this work, considering different simulation techniques, system sizes and thermophysical properties. The workflow for the development and optimization of force field models, which are the basis of every molecular simulation, was presented. Three new force field models for cyclohexamine, aniline and phenol were parametrized and validated against experimental VLE data.

As an application for large scale MD simulations, the evaporation of liquid nitrogen into a supercritical hydrogen atmosphere was presented. Here, special attention was paid to the behavior of the interface between the two phases. Temperature, density and concentration profiles were sampled to study the dependencies of the evaporation flux.

Further, different thermophysical properties of mixtures were predicted. Kirkwood-Buff integrals, which are related to the radial distribution functions and describe local density fluctuations, were used to sample the thermodynamic factor of binary LJ mixtures. Because the results show a significant system size dependence, a good scaling behavior of the simulation software is necessary, which is shown to be given for the utilized simulation package $m s 2$. The temperature dependent Henry's 

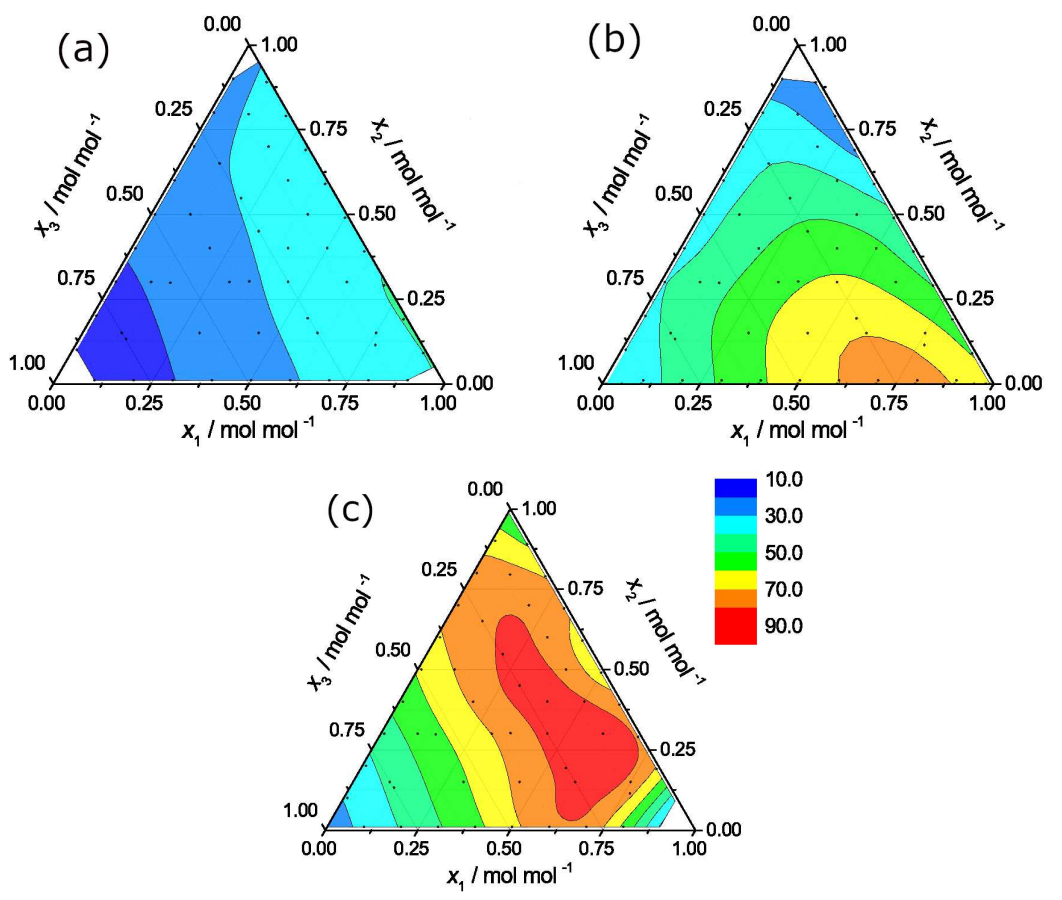

Fig. 9 Maxwell-Stefan diffusion coefficients $\bigoplus_{12}$ (a), $\bigoplus_{13}$ (b) and $\bigoplus_{23}$ (c) (in $10^{-10} \mathrm{~m}^{2} \mathrm{~s}^{-1}$ ) of acetone (1) + benzene (2) + methanol (3) at $T=298.15 \mathrm{~K}$ and $p=0.1 \mathrm{MPa}$.

law constant of aqueous systems containing $\mathrm{H}_{2}, \mathrm{~N}_{2}, \mathrm{O}_{2}$ or $\mathrm{Ar}$ was calculated from chemical potential data sampled with Widom's test particle insertion. The results are in excellent agreement with experimental data. Finally, results for the intradiffusion and Maxwell-Stefan diffusion coefficients were presented for the ternary mixture acetone + benzene + methanol in the liquid state covering the entire composition range of the given mixture.

Acknowledgements We gratefully acknowledge support by Deutsche Forschungsgemeinschaft. This work was carried out under the auspices of the Boltzmann-Zuse Society (BZS) of Computational Molecular Engineering. The simulations were performed on the CRAY XC40 (Hazel Hen) at the High Performance Computing Center Stuttgart (HLRS) within the project MMHBF2.

\section{References}

1. Deublein, S., Eckl, B., Stoll, J., Lishchuk, S. V., Guevara-Carrion, G., Glass, C. W., Merker, T., Bernreuther, M., Hasse, H., Vrabec, J.: ms2: A molecular simulation tool for thermodynamic properties. Comput. Phys. Commun. 182, 2350-2367 (2011) 
2. Glass, C. W., Reiser, S., Rutkai, G., Deublein, S., Köster, A., Guevara-Carrion, G., Wafai, A., Horsch, M., Bernreuther, M., Windmann, T., Hasse, H., Vrabec, J.: ms2: A molecular simulation tool for thermodynamic properties, new version release. Comp. Phys. Commun. 185, 3302-3306 (2014)

3. Rutkai, G., Köster, A., Guevara-Carrion, G., Janzen, T., Schappals, M., Glass, C. W., Bernreuther, M., Wafai, A., Stephan, S., Kohns, M., Reiser, S., Deublein, S., Horsch, M., Hasse, H., Vrabec, J.: ms2: A molecular simulation tool for thermodynamic properties, release 3.0. Comp. Phys. Commun. 221, 343-351 (2017)

4. Niethammer, C., Becker, S., Bernreuther, M., Buchholz, M., Eckhardt, W., Heinecke, A., Werth, S., Bungartz, H.-J., Glass, C. W., Hasse, H., Vrabec, J., Horsch, M.: 1s1 mardyn: The massively parallel molecular dynamics code for large systems. J. Chem. Theory Comput. 10, 4455-4464 (2014)

5. Chmiela, S., Tkatchenko, A., Sauceda, H. E., Poltavsky, I., Schütt, K. T., Müller, K.-R.: Machine learning of accurate energy-conserving molecular force fields. Science Advances 3, 1-6 (2017)

6. Li, Y., Li, H., Pickard, F. C., Narayanan, B., Sen, F. G., Chan, M. K. Y., Sankaranarayanan, S. K. R. S., Brooks, B. R., Roux B.: Machine learning force field parameters from ab initio data. J. Chem. Theory Comput. 13, 4492-4503 (2017)

7. Ho, Y.-C., Wang, Y.-S., Chao, S. D.: Molecular dynamics simulations of fluid cyclopropane with MP2/CBS-fitted intermolecular interaction potentials. J. Chem. Phys. 147, 064507, (2017)

8. Nezbeda, I.: Simulations of vaporliquid equilibria: Routine versus thoroughness. J. Chem. Eng. Data 61, 3964-3969 (2016)

9. Dahms, R., and Oefelein, J. C.: On the transition between two-phase and single-phase interface dynamics in multicomponent fluids at supercritical pressures. Phys. Fluids 25, 092103 (2013)

10. Heinen, M., Vrabec, J., and Fischer, J., J.: Communication: Evaporation: Influence of heat transport in the liquid on the interface temperature and the particle flux. Chem. Phys. 145, 081101 (2016)

11. Köster, A., Thol, M., Vrabec, J.: Molecular Models for the Hydrogen Age: Hydrogen, Nitrogen, Oxygen, Argon, and Water. J. Chem. Eng. Data 63, 305-320 (2018)

12. Galata, A. A., Anogiannakis, S. D., Theodorou, D. N.: Thermodynamic analysis of LennardJones binary mixtures using Kirkwood-Buff Theory. Fluid Phase Equilib. 470, 25-37 (2018)

13. Gross, J., Sadowski, G.: Perturbed-chain SAFT: An equation of state based on a perturbation theory for chain molecules. Ind Eng Chem Res. 40, 1244-1260 (2001)

14. Kirkwood, J. G., Buff, F.P.: The Statistical Mechanical Theory of Solutions. I. J. Chem. Phys. 19, 774-778 (1951)

15. Milzetti, J., Nayar, D., van der Vegt, N. F. A.: Convergence of Kirkwood-Buff Integrals of Ideal and Nonideal Aqueous Solutions Using Molecular Dynamics Simulations. J. Phys. Chem. B 122(21), 5515-5526 (2018)

16. KrÃijger, P.,Schnell, S. K.,Bedeaux, D., Kjelstrup, S., Vlugt, T. J. H., Simon, J. M.: Kirkwood-Buff integrals for finite volumes. J. Phys. Chem. Lett. 4, 10911-10918 (2013) 235 âĂŞ238.

17. Schnell, S. K., Liu, X., Simon, J.-M., Bardow, A., Bedeaux, D., Vlugt, T. J. H., Kjelstrup, S.: Calculating Thermodynamic Properties from Fluctuations at Small Scales. J. Phys. Chem. B 115, 10911-10918 (2011)

18. Widom, B.: Some Topics in the Theory of Fluids. J. Chem. Phys. 39, 2808-2812 (1963)

19. Wilson, G. M.: Vapor-liquid equilibrium. XI. A new expression for the excess free energy of mixing. J. Am. Chem. Soc. 86, 127 (1964)

20. Shing, K.S., Gubbins, K. E., Lucas K.: Henry constants in non-ideal fluid mixtures: computer simulation and theory. Mol. Phys. 65, 1235-1252 (1988)

21. Fernández-Prini, R., Alvarez, J. L., Harvey, A. H.: Henry's constants and vapor-liquid distribution constants for gaseous solutes in $\mathrm{H}_{2} \mathrm{O}$ and $\mathrm{D}_{2} \mathrm{O}$ at high temperatures. J. Phys. Chem. Ref. Data 32, 903-916 (2003) 
22. International Association for the Properties of Water and Steam. Guideline on the Henry's Constant and Vapor-Liquid Distribution Constant for Gases in $\mathrm{H}_{2} \mathrm{O}$ and $\mathrm{D}_{2} \mathrm{O}$ at High Temperatures (2004)

23. Morris, D. R., Yang, L., Giraudeau, F., Sun, X., Steward, F. R.: Henry's law constant for hydrogen in natural water and deuterium in heavy water. Phys. Chem. Chem. Phys. 3, 1043$1046(2001)$

24. Alvarez, J., Fernández-Prini, R.: A semiempirical procedure to describe the thermodynamics of dissolution of non-polar gases in water. Fluid Phase Equilib. 66, 309-326 (1991)

25. Rettich, T. R., Battino, R., Wilhelm, E.: Solubility of gases in liquids. XVI. Henry's law coefficients for nitrogen in water at 5 to $50^{\circ} \mathrm{C}$. J. Solution Chem. 13, 335-348 (1984)

26. Cosgrove, B. A., Walkley, J.: Solubilities of gases in $\mathrm{H}_{2} \mathrm{O}$ and ${ }^{2} \mathrm{H}_{2} \mathrm{O}$. J. Chromatogr. A 216, 161-167 (1981)

27. Alvarez, J., Crovetto, R., Fernández-Prini, R.: The dissolution of $\mathrm{N}_{2}$ and of $\mathrm{H}_{2}$ in water from room temperature to 640 K. Ber. Bunsenges. Phys. Chem. 92, 935-940 (1988)

28. Rettich, T. R., Battino, R., Wilhelm, E.: Solubility of gases in liquids. 22. High-precision determination of Henry's law constants of oxygen in liquid water from $\mathrm{T}=274 \mathrm{~K}$ to $\mathrm{T}=328$ K. J. Chem. Thermodyn. 32, 1145-1156 (2000)

29. Benson, B. B., Krause, D., Peterson, M. A.: The solubility and isotopic fractionation of gases in dilute aqueous solution. I. Oxygen. J. Solution Chem. 8, 655-690 (1979)

30. Rettich, T. R., Handa, Y. P., Battino, R., Wilhelm, E.: Solubility of gases in liquids. 13. Highprecision determination of Henry's constants for methane and ethane in liquid water at 275 to 328 K. J. Phys. Chem. 85, 3230-3237 (1981)

31. Cramer, S. D: The solubility of oxygen in brines from 0 to $300^{\circ} \mathrm{C}$. Ind. Eng. Chem. Process Des. Dev. 19, 300-305 (1980)

32. Rettich, T. R., Battino, R., Wilhelm, E.: Solubility of gases in liquids. 18. High-precision determination of Henry fugacities for argon in liquid water at 2 to $40^{\circ} \mathrm{C}$. J. Solution Chem. 21, 987-1004 (1992)

33. Krause, D., Benson, B. B.: The solubility and isotopic fractionation of gases in dilute aqueous solution. IIa. Solubilities of the noble gases. J. Solution Chem. 18, 823-873 (1989)

34. Potter, R. W., Clynne, M. A.: The solubility of the noble gases $\mathrm{He}, \mathrm{Ne}, \mathrm{Ar}, \mathrm{Kr}$, and $\mathrm{Xe}$ in water up to the critical point. J. Solution Chem. 7, 837-844 (1978)

35. Crovetto, R., Fernández-Prini, R., Japas, M. A.: Solubilities of inert gases and methane in $\mathrm{H}_{2} \mathrm{O}$ and in $\mathrm{D}_{2} \mathrm{O}$ in the temperature range of 300 to $600 \mathrm{~K}$. J. Chem. Phys. 76, 1077-1086 (1982)

36. Allen, M.P., Tildesley, D.J.: Computer simulation of liquids. Clarendon Press, Oxford (1987)

37. Krishna, R., van Baten, J.M.: The darken relation for multicomponent diffusion in liquid mixtures of linear alkanes: an investigation using molecular dynamics (MD) simulations. Ind. Eng. Chem. Res. 44, 6939-6847 (2005)

38. Guevara-Carrion, G., Gaponenko, Y., Janzen, T., Vrabec, J., Shevtsova, V.: Diffusion in Multicomponent Liquids: From Microscopic to Macroscopic Scales. J. Phys. Chem. B 120, $12193-$ 12210 (2016)

39. Janzen, T., Gaponenko, Y., Mialdun, A., Guevara-Carrion, G., Vrabec, J., Shevtsova V.: The effect of alcohols as the third component on diffusion in mixtures of aromatics and ketones. RSC Adv. 8, 10017-10022 (2018)

40. Guevara-Carrion, G., Janzen, T., Muñoz-Muñoz, Y. M., Vrabec, J.: Molecular Simulation Study of Transport Properties for 20 Binary Liquid Mixtures and New Force Fields for Benzene, Toluene and CCl4. In: Nagel, W., Kröner, D., Resch, M. (eds) High Performance Computing in Science and Engineering '16. Springer, Cham (2016)

41. Alimadadian, A., and C. Phillip Colver: A new technique for the measurement of ternary molecular diffusion coefficients in liquid systems. Can. J. Chem. Eng. 54(3), 208-213 (1976)

42. Taylor, R., Krishna, R.: Multicomponent mass transfer. John Wiley and Sons, New York (1993) 